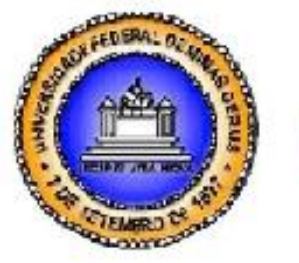

\title{
SOFTWARE PARA PREVENÇÃO DE BURNOUT NO USO DE EQUIPAMENTOS MÉDICOS: UMA PROPOSTA PARA DETECÇÃO DE INDÍCIOS DE ESTRESSE EM CENTROS CIRÚRGICOS E INTENSIVOS
}

\author{
SOFTWARE FOR PREVENTION OF BURNOUT IN USE OF MEDICAL \\ EQUIPMENT: A PROPOSAL FOR THE DETECTION OF EVIDENCE OF STRESS IN \\ WORKERS OF SURGICAL AND INTENSIVE UNITS
}

SOFTWARE PARA LA PREVENCIÓN DE BURNOUT EN USO DE EQUIPO MÉDICO: UNA PROPUESTA PARA LA DETECCIÓN DE PRUEBAS DE ESTRÉS EN LOS TRABAJADORES DE LAS UNIDADES DE CIRUGÍA Y INTENSIVOS

\begin{tabular}{|l|l|}
\hline $\begin{array}{l}\text { Daniel Gomes de Moura } \\
\text { Universidade FUMEC } \\
\text { danielllgm@gmail.com }\end{array}$ & Submetido em: 24/10/2015 \\
$\begin{array}{l}\text { Daniel Jardim Pardini } \\
\begin{array}{l}\text { Universidade FUMEC } \\
\text { pardini@fumec.br }\end{array}\end{array}$ & \\
\hline & DOI 10.21450/rahis.v13i3.3799 \\
\hline
\end{tabular}

Resumo: Quando a cultura organizacional de um hospital afeta os profissionais de saúde de forma negativa, a segurança do paciente não pode ser garantida. Tal fato se deve ao esforço diário desses trabalhadores, que acabam por esgotar precocemente sua energia produtiva ao buscarem soluções simplificadas e transitórias para problemas sistêmicos com que se debatem. Assim, ao longo da jornada de trabalho, tais profissionais experimentam os efeitos do estresse e convivem com erros humanos provocados pelo esgotamento físico e mental. Tal esgotamento repercute também em defeitos na interface com os equipamentos médicos. Dessa forma, este trabalho propõe um modelo em software para detectar os indícios do estresse em busca de prevenção da Síndrome de Burnout através da investigação dos registros de manutenção, ao mesmo tempo que questiona a eficácia da reaplicação de treinamento em equipamentos médicos. Portanto, através da aplicação de conceitos psicológicos e de engenharia, o ensaio do software considerou os históricos de manutenção em dois hospitais brasileiros. O software mostrou-se promissor não só como medida de prevenção da doença, mas como indicador de qualidade dos serviços de saúde.

Palavras-chave: Burnout. Software. Segurança do paciente. 


\begin{abstract}
When the organizational culture of a hospital affects health professionals negatively, the patient safety can not be guaranteed. This is due to the daily effort of these workers, who prematurely exhaust their productive power in the search of simplified and transitional solutions to systemic problems they face. Thus, throughout the working week, these professionals have experienced the effects of stress: the human errors caused by physical and mental exhaustion. Such effect has reflected in defects at the interface with medical equipment too. Thus, this text proposes a model of software to detect the evidence of stress seeking to prevent burnout syndrome through research in maintenance, while it questioning the effectiveness of repeating training in medical equipment. Therefore, by applying the concepts of psychology and engineering, the software test has considered the historical of maintenance in two Brazilian hospitals. The software has shown itself as a promise not only as a measure to prevent the disease, but as a quality indicator of health services.
\end{abstract}

Keywords: Burnout. Software. Patient safety. 
Resumen: Cuando la cultura organizacional de un hospital afecta a profesionales de la salud negativamente, la seguridad de los pacientes no se puede garantizar. Esto se debe al esfuerzo diario de estos trabajadores, que antes de tiempo agota su fuerza productiva en la búsqueda de soluciones simplificadas y de transición a los problemas sistémicos que enfrentan. Por lo tanto, durante toda la semana de trabajo, estos profesionales han experimentado los efectos del estrés: los errores humanos causados por el agotamiento físico y mental. Dicho efecto se ha reflejado en los defectos en la interfaz con el equipo médico también. Por lo tanto, este texto propone un modelo de software para detectar la evidencia de estrés tratando de prevenir el síndrome de burnout través de la investigación en el mantenimiento, al tiempo que cuestiona la eficacia de la repetición de la formación en equipos médicos. Por lo tanto, mediante la aplicación de los conceptos de la psicología y la ingeniería, la prueba de software ha considerado el histórico de mantenimiento en dos hospitales brasileños. El software se ha mostrado como una promesa no sólo como una medida para prevenir la enfermedad, sino como un indicador de la calidad de los servicios de salud..

Palabras-clave: Burnout. Software. Seguridad del paciente. 


\section{INTRODUÇÃO}

O Ministério da Saúde tem realizado diversos projetos focados no desenvolvimento do Sistema Único de Saúde (SUS) ao longo dos últimos anos. São exemplos os programas REFORSUS, SOMASUS e, mais recentemente, o programa Mais Médicos. Contudo, com certa frequência, surgem nos meios populares de comunicação queixas de atendimento, feitas por pacientes e seus familiares. Por outro lado, os profissionais de medicina e enfermagem, também conhecidos como profissionais de assistência e que atuam diretamente com os pacientes, rebatem e justificam a situação citando que as condições de trabalho e infraestrutura são inadequadas ou situam-se em precariedade frente ao objetivo de prover saúde gratuita de qualidade para a população.

Implícito no cenário encontra-se a tecnodependência na prestação de serviços de saúde. Assim, o avanço tecnológico, tendo como força motriz a competição por mercados, trouxe consigo, ao mesmo tempo, vantagens e riscos quanto à aplicação de equipamentos médicos junto aos pacientes e aos profissionais de saúde. Erros de operação dos equipamentos médicos podem ocorrer na interface homem-máquina, sendo alguns desses erros motivados por fatores psicológicos, como a desatenção ou a falha de memória dos profissionais de saúde que os operam. Tais fatores psicológicos podem ser fruto do ambiente laboral, como apontam os profissionais de assistência, resultando em dano ou morte do paciente, por um lado, e/ou em manutenções corretivas dos equipamentos médicos, por outro.

Ainda sobre o aspecto psicológico, estudos indicam que parte dos profissionais não está em condições de atuar de modo satisfatório, agindo frequentemente com distanciamento intelectual de suas atividades laborais. Esse distanciamento caracteriza-se por meio da adoção de uma postura autômata e aparentemente desprovida de envolvimento emocional. Tal fenômeno tem recebido o nome de Síndrome de Burnout e manifesta-se pela cronificação do estresse no trabalho, resultando em esgotamento físico e mental. Durante seu desenvolvimento, o estresse grave favorece o aumento de risco de erros de operação da tecnologia por desatenção, falhas de memória e dificuldades de raciocínio. Em certos casos, sendo a cultura organizacional voltada para a culpabilização, fomenta entre os profissionais de assistência o desgaste interpessoal e falhas de comunicação, comprometendo o clima de trabalho em equipe e proporcionando aumento de acidentes, absenteísmo e abandono da profissão. Para o paciente implica maior risco de dano físico ou morte, posto que erros humanos possam resultar em eventos adversos. Para as organizações, implica perda financeira e de produtividade pela necessidade de treinar periodicamente novos profissionais.

Dentro do hospital, os centros de terapia intensiva (CTI) e os centros cirúrgicos (CC) representam locais de interesse para o estudo do desenvolvimento do estresse em profissionais de saúde, uma vez que o tratamento do paciente demanda maior envolvimento da equipe assistencial na tomada rápida de decisões. Também são locais com grande concentração de equipamentos médicos e de profissionais, sejam estes professores (preceptores) ou alunos (residentes), envoltos na prática da medicina. A combinação descuidada desses elementos sob a cultura organizacional pode resultar em erros e ou em eventos adversos.

No passado recente, a aplicação de investigações para tratar a causa-raiz dos erros permitiu a evolução de medidas de segurança, como por exemplo, com relação aos equipamentos de anestesia usados dentro do centro cirúrgico. Para Martins e Fragata (2014, p. 107), a anestesiologia é a especialidade médica que mais investe na segurança, sendo o caso de maior sucesso na área da medicina. São exemplos de mudanças adotadas contra a falha de memória e de lapsos a aplicação 
de código de cores distintas para os agentes anestésicos e de encaixes diferentes na interface homem-máquina, de tal modo que, mesmo que o profissional não tivesse prestado a devida atenção nas cores, não conseguiria conectar erradamente o equipamento médico e provocar aplicação indevida de anestesia ao paciente ao longo do ato cirúrgico. Porém, existem casos de interface homem-máquina que ainda estão em processo de amadurecimento quanto à gestão de riscos, como é o caso da bomba de infusão nos centros de terapia intensiva. Injeções incorretas de medicamentos ou de doses erradas frente à prescrição médica, com posterior dano ou morte, têm sido reportadas pela mídia com certa frequência.

Onde a evolução da tecnologia ainda não foi capaz de prover um nível de segurança adequado, o profissional de saúde precisa identificar pontos de melhoria nos procedimentos operacionais e motivar soluções dentro da equipe para a prevenção do risco de eventos adversos, atuando no ambiente de trabalho, de modo a maximizar o aproveitamento dos investimentos na melhoria do Sistema Único de Saúde brasileiro. Essa atuação deve ser mais ampla, com vistas aos fatores humanos como a carga e a jornada de trabalho, por exemplo, e não pode ser simplificada em reaplicação de treinamentos de uso dos equipamentos médicos ou na política da culpabilização dos erros.

O objetivo do presente artigo é propor protótipo de avaliação indireta, silenciosa e retroativa de indícios de estresse e de síndrome de Burnout por meio dos erros de operação cometidos pela equipe assistencial junto a seus equipamentos médicos em centros cirúrgicos e de terapia intensiva. O protótipo de avaliação será através de software capaz de processar os registros de manutenção em busca de tais indícios dentro de um sistema de gerenciamento utilizado pela engenharia clínica, sendo considerados como exemplos de aplicação casos de equipamentos médicos de anestesia e de bombas de infusão. Os registros analisados constituem históricos de manutenção de dois hospitais do estado de Minas Gerais, geograficamente distantes entre si, entre os anos de 2012 e 2014.

\section{SÍNDROME DE BURNOUT}

Burn-out é um termo antigo e no jargão popular inglês se refere ao que deixou de funcionar por absoluta falta de energia, sendo que a maioria dos autores indica Herbert J. Freudenberger como o primeiro a utilizar o termo em seu artigo "Staff Burn-out", datado de 1974, para alertar a comunidade científica dos problemas a que os profissionais de saúde estão expostos em função de seu trabalho (PEREIRA, 2014, p. 30). Para Pereira (2014, p. 45), o Burnout também pode ser entendido como a resposta a um estado prolongado de estresse e ocorre pela cronificação deste. Sua natureza pode ser entendida como sendo uma experiência individual específica do contexto de trabalho (MASLACH; SCHAUFELI; LEITER, 2001, p. 407).

Maslach e Jackson (1986) descreveram o Burnout como composto por três dimensões básicas: a exaustão emocional (EE), que é a dimensão central da síndrome e se caracteriza pela sensação de não mais dispor de nenhum resquício de energia física ou mental, trazendo uma série de sintomas psicossomáticos que acarretam absenteísmo e afastamento por problemas de saúde; a despersonalização (DE), que representa a dimensão defensiva aos sintomas e se caracteriza por atitudes desumanizadas, irônicas e cínicas para com os demais; e a reduzida realização profissional (rRP), que se caracteriza pelo sentimento de frustação, insatisfação pessoal e auto avaliação negativa em lugar do idealismo e da motivação do início do trabalho (PEREIRA, 2014, p. 35).

Considerando o trabalho de Maslach e Jackson, a maioria dos pesquisadores utiliza o Maslach Burnout Inventory (MBI) para demonstrar academicamente a presença da síndrome de Burnout entre os profissionais de assistência no Brasil. Lima et al. (2007, p. 145) constata que o 
período de residência médica com suas características de dualidade de papéis (aprendizagem e trabalho) e grande exigência constituem uma fase propícia à instalação da Síndrome de Burnout. Em relação à enfermagem, Pinho (2002) apud Aquino (2005, p. 40) ressalta que as enfermeiras que trabalham em centros cirúrgicos são, normalmente, envolvidas por estresse, tensão emocional e outras dificuldades que as sobrecarregam de forma física e psicológica, devido à ansiedade produzida pelo ato cirúrgico gerada na equipe e a fatores que podem surgir diante de situações imprevistas. Pafaro e Martino (2004, p. 157) constataram, por sua vez, que $70,84 \%$ dos profissionais de saúde que tinham dupla jornada de trabalho em busca do complemento de renda familiar apresentavam sinais de estresse através da aplicação de Inventário de Sintomas de Stress de Lipp (ISSL) e de Escala Analógica Visual (EAV), sendo este número consideravelmente maior que o percentual de profissionais que trabalhavam em uma única jornada.

Desta forma, há a preocupação em conciliar descanso e trabalho. Pereira (2014, p. 144) cita Westiman e Éden (1997) quanto à confirmação de que quanto maior o tempo de descanso, menor a EE. Martins e Fragata (2014, p. 132) apontam que aspectos tão fundamentais como a fadiga, o stress, a incapacidade de liderança e a incapacidade de funcionar como uma equipe são de enorme importância e representam condições predisponentes ao erro em medicina. Santos (2011) cita que um profissional que está burning-out tende a criticar tudo e todos os que o cercam e tem pouca energia para as diferentes solicitações de seu trabalho, o que compromete o clima necessário para o trabalho em equipe. Já Ferreira (2000) aponta que o elevado nível de estresse pode refletir em atrasos, insatisfação, sabotagem e baixos níveis de desempenho no trabalho.

Logo, o esforço de lidar com a jornada de trabalho pode ser apreciado através de critérios usados na elaboração de escalas de trabalho. Poltosi e Gómez (2007), em seu modelamento computacional para resolver o problema do trato manual da escala de trabalho, basearam-se nos estudos de Burke et al. (2004) e consideraram como desejável ao processamento o respeito às solicitações de datas por parte dos profissionais de saúde, a distribuição de plantões, os finais de semana e as folgas adicionais. Portanto, a escala de trabalho visa obter o maior nível de satisfação dos funcionários, atender as preferências de folgas e a distribuição justa de plantões impopulares, respeitando a legislação e as normas vigentes.

\section{SEGURANÇA DO PACIENTE}

Para a Organização Mundial de Saúde (OMS), segurança do paciente corresponde à redução ao mínimo aceitável do risco de dano desnecessário associado ao cuidado de saúde (ANVISA, 2013, p. 24). Assim, Martins e Fragata (2014, p. 373) caracterizam o evento adverso como sendo todo o efeito não desejado que resulte da intervenção dos cuidados de saúde ou de sua falta. Portanto, o gerenciamento de riscos (Risk Management) tem focado problemas de segurança do paciente devido aos seus riscos associados, principalmente os financeiros, para as organizações de saúde e seu pessoal, derivados fundamentalmente de litígios por condutas erradas. Seu enfoque é predominantemente prospectivo e preventivo, embora geralmente esteja baseado em problemas detectados anteriormente. Dada a sua importância, a integração das atividades desenvolvidas pela gerência de riscos com outras metodologias (como, por exemplo, a ABNT ISO 31000:2009) tem sido identificada como um dos sete passos essenciais para a segurança do paciente, segundo a Agência Nacional de Segurança do Paciente (National Patient Safety Agency - NPSA), do Reino Unido (ANVISA, 2013, p. 34).

Contudo, Reason (1995) cita que o comportamento humano, para o bem ou para o mal, claramente predomina frente aos sistemas tecnológicos modernos, sejam estes sistemas médicos ou 
quaisquer outros, por que são os humanos que os operam, os constroem, os projetam, os mantêm e os organizam. Todas as contribuições humanas já foram englobadas através do termo "erro humano", contudo, os atos inseguros vêm assumindo diferentes formas: deslizes, lapsos e enganos, erros e violações - cada um com diferentes origens psicológicas e com necessidades de diferentes contramedidas (REASON, 1995).

Reason (1995) explica ainda que as falhas podem ser ativas ou latentes. Quando ativas ocasionam resultados negativos imediatos, como por exemplo, erros de "ponta de bisturi" dentro do sistema, que foram cometidos por cirurgiões, anestesistas, enfermeiros e fisiologistas, etc. junto aos pacientes, resultando em eventos adversos. Quando latentes, são resultado de decisões tomadas pelo alto escalão de uma organização, sendo que suas consequências podem ficar adormecidas por longos anos até finalmente virem à tona devido a fatores catalisadores de violação das defesas do sistema.

Assim, como meio de identificar vulnerabilidades na assistência ao paciente, em 1998, o National Center for Patient Safety (NCPS - Centro Nacional para Segurança ao Paciente), do U.S. Department of Veterans Affairs (VA), instituiu o Programa de Segurança ao Paciente e, em 2001, após examinar vários modelos analíticos proativos utilizados pela indústria, desenvolveu um método de análise prospectiva híbrido, considerando o FMEA e denominado Health Care Mode Effect Analysis (HFMEA) (DeROSIER et al., 2002; VA-NCPS, 2001 apud GLOWACKI, 2003, p. 54). Para Martins e Fragata (2014, p. 196), o HFMEA serve de instrumento para a detecção de disfunções capazes de serem minorados, mas sem o questionamento dos pressupostos em que o sistema atua, e referenciam Tucker e Edmondson (2003), ao caracterizar a aplicação deste modelo como sendo de esforço de resolução de problemas que surgiam em single loop, ou seja, capaz de produzir satisfação pela eficácia alcançada na resolução desses, por um lado, e o esgotamento (Burnout) que essa postura permanente provoca, por outro. Portanto, o HFMEA se mostra limitado por não diagnosticar os problemas de segunda ordem, ou em double loop.

Wu e Zhao (2011) apontam outra limitação comum quanto à análise de risco ao explicar que embora existam diversas técnicas, geralmente elas investem pouca atenção ao software ou aos fatores humanos, e que a relação de interdependência entre eles dificilmente poderia ser tratada. Ainda, explicam que o principal propósito de se analisar a confiabilidade humana é prover uma probabilidade crível sobre o erro humano, porém, diante de sua diversidade e alta complexidade, não existe análise de confiabilidade aplicável ao seu comportamento, sendo esta a regra intrínseca ao sistema complexo. Ainda, os autores citam que em tais sistemas, como a aviação, a interação homem-máquina é a principal razão de acidentes. Para Martins e Fragata (2014, p. 112), o hospital também consiste em sistema complexo, e citam que "pilotos em seus cockpits podem ser comparados a anestesistas em seus centros operatórios".

Ainda em relação à aviação, em busca de nova abordagem quanto à interação homemmáquina que pudesse melhor explicar acidentes, Wu e Zhao (2011) apresentam modelo de gestão de risco denominado Complex Human Factor Analysis and Classification Framework (C-HFACF) considerando a teoria de acidente baseada em energia (energy-based accident causation theory), segundo a qual, quando o ser humano necessita usar sua energia, esta deve ser medida para ser produzida, controlada e transformada de tal forma que possa realizar o trabalho de acordo com a intenção daquela pessoa. A falta ou o excesso acidental de liberação de energia pode resultar em algo indesejado, danificar o corpo, assim como o equipamento e o ambiente do entorno ao mesmo (WU; ZHAO, 2011).

Martins e Fragata (2014, p. 54) citam, por sua vez, que de fato pacientes e equipamentos podem sofrer danos por conta de erros em um sistema complexo. Historicamente, Shepherd et al. 
(1976) apresentou modelo de gestão de risco que mais tarde serviria de base para a educação de enfermagem, evoluindo em 1997 para receber o nome Systems Risk Model (SRM). Em 2004, o SRM já era representado por um envelope que poderia ilustrar o provedor de serviços de saúde (hospital, clínica, homecare, etc.), sendo que no centro do envelope encontra-se uma área sombreada, contendo os termos Operator, Facility, Device, Environment e Patient, representando assim um minissistema genérico capaz de fornecer o benefício clínico desejado no final da cadeia de cuidados ao paciente. Cada um dos cinco componentes representa por sua vez envelopes ainda menores, e cada um deles contém um conjunto característico de subcomponentes. A falha encontrada no interior de cada subcomponente dentro do envelope pode conduzir à falha do componente em si, o que poderia resultar em evento adverso (DYRO, 2004).

SÁ (2009, p. 17) aponta que em 2006 o SRM foi alterado de 14 para 16 subcomponentes, conforme artigo do ACCE - Healthcare Technology Foundation, sendo que a revisão atingiu o componente Operator, expandindo as subcomponentes em Educação/treinamento, Erro de uso (uso não pretendido), Desatenção (Falta de atenção) e Uso intencional (criminal).

Amoore (2014), ao propor seu próprio modelo de gestão de risco, compara a versão revisada do SRM aos modelos de classificação do Emergency Care Research Institute (ECRI) e ao modelo hierárquico de classificação de causas, adotado pela International Standards Organizations (ISO), e decide manter como falhas relacionadas ao operador a distração e a fadiga, bem como o abuso malicioso.

Portanto, a gestão de risco a partir da manutenção, através do componente Operator, considera causas também relacionadas aos sintomas psíquicos citados por Pereira $(2014$, p. 44$)$ tais como falta de atenção, de concentração e alterações de memória; aos sintomas físicos de fadiga constante e progressiva e aos sintomas comportamentais como negligência e comportamento de risco. A síntese gráfica que ilustra como os indícios de estresse podem ter sido coletados a partir da gestão da manutenção de equipamentos médicos está representada pela figura 1, na qual o operador, após perceber uma falha no equipamento médico, age de imediato através de uma ordem de serviço (OS) de manutenção corretiva (CALIL; GOMIDE, 2002, p. 72).

FIGURA 1 - Síntese gráfica

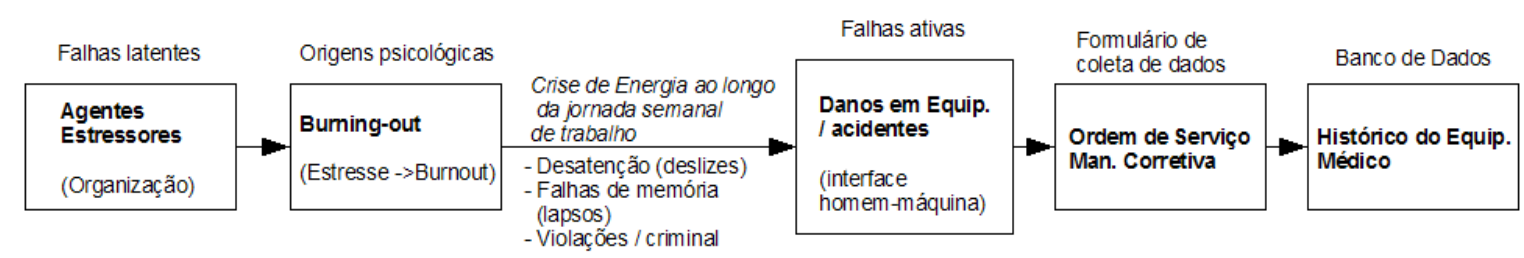

Fonte: Elaborado pelos autores.

\section{PROTÓTIPO DE SOFTWARE}

A frequência com que o profissional de saúde em burning-out recorre, consciente ou inconscientemente, a interrupção das atividades laborais tendo por justificativa a tecnodependência, ou seja, a indisponibilidade de equipamento médico pode ser obtida através da análise dos registros do histórico de manutenção. Seguindo o exemplo de modelos de gestão de risco como o SRM, o protótipo de software proposto deve ser capaz de classificar os registros existentes no histórico, a fim de determinar quais tiveram o componente Operator como causa raiz. Assim, considerando 
técnicas de engenharia de software, como, por exemplo, o uso da linguagem artificial de pseudocódigos apresentada por Deitel e Deitel (1999, p.47), o SRM sugerido por Shepherd pôde ser reordenado no código-fonte, resultando no fluxograma da figura 2.

O protótipo faz a consulta aos registros do sistema de informação em engenharia clínica do hospital. Assim, por exemplo, na etapa 4 (Ambiente), a ocorrência de ordens de serviço contemporâneas para a manutenção corretiva predial de infraestrutura hospitalar é submetida a análise que contabiliza os registros que apontem serviços de manutenção dentro do setor assistencial que possam ter influenciado o operador, como obras de alvenaria (que podem provocar agentes estressores como ruído e cheiro de tinta, por exemplo), solicitação de substituição de lâmpadas, danos em eletrodomésticos e alterações em quadros de energia que alimentam os equipamentos médicos, dentre outros (ELIAS, 2013).

De forma análoga, as demais etapas ocorrem de acordo com o ciclo de vida do equipamento médico até que, por eliminação, o registro de falha seja creditado ao componente Operator (Operador). O ciclo de vida de um equipamento médico inicia-se com o planejamento de sua incorporação (HERMINI, 2013, p. 133), seguida da aquisição, entrega e inspeção, instalação e aceitação, treinamento de usuários e operadores, monitoramento de uso e desempenho, manutenção, e encerra-se com a troca ou descarte (DYRO, 2004, p. 129). Desta forma, a etapa 5 do fluxograma, chamada de instalação, compreende do planejamento ao treinamento de usuários e operadores. Também nesta etapa, considera-se a curva de aprendizagem limitada pelo tempo da garantia de fábrica do equipamento médico, chamada neste estudo de inicialização, ou seja, falhas que ocorrem enquanto há o suporte técnico vinculado ao fornecimento e que são fruto do esforço para adequar novas técnicas aos procedimentos dos operadores (MARTINS; FRAGATA, 2014, p. 313). A etapa 6, por sua vez, compreende a parte restante do ciclo de vida do equipamento médico, quando este está no ápice de sua produção. A etapa 6 abrange ainda o monitoramento de uso e performance, a manutenção e, eventualmente, a necessidade de renovação por fïm de vida útil (com consequente troca ou descarte do equipamento médico). É nesta etapa que se verifica também se a falha ocorreu devido a desgaste natural de componente interno que necessite do uso de ferramentas para a troca e, portanto, fora da interface homem-máquina (HERMINI, 2013, p. 168).

$\mathrm{Na}$ etapa 7, os registros de manutenção corretiva que foram atribuídos ao componente Operator são contabilizados e nova classificação pode ser implementada a fim de verificar a distribuição de ocorrências de falhas em equipamentos médicos ao longo da jornada de trabalho semanal do operador. Desta forma, considerando a teoria de acidente baseada em energia citada por Wu e Zhao (2011), bem como a necessidade de conciliar descanso e trabalho como medida necessária à prevenção do agravamento da componente central $\mathrm{EE}$ da síndrome de Burnout, conforme citado por Pereira (2014, p. 144), e tendo em vista que a própria definição do termo Burnout aponta para o esgotamento da energia para as atividades laborais, sendo esta proporcional ao tempo de jornada e à carga de trabalho realizada pelo operador (PEREIRA, 2014, p. 133), tornase possível especular sobre o ciclo semanal de energia do operador como sendo representado por um indicador gráfico de carga de bateria conforme figura 3. Para uma semana sem feriados, o sábado é o dia no qual se inicia o descanso e será representado pela letra E (energia ou evento). Assim, para indicar o nível de energia e para mostrar, em dias, a antecedência em relação à E, cada dia útil é indexado de E-1 (sexta-feira, onde ocorre o nível mais baixo de energia) à E-6 (domingo, quando a energia foi reestabelecida para o nível mais alto). 
FIGURA 2 - Fluxograma simplificado para classificação baseada em SRM

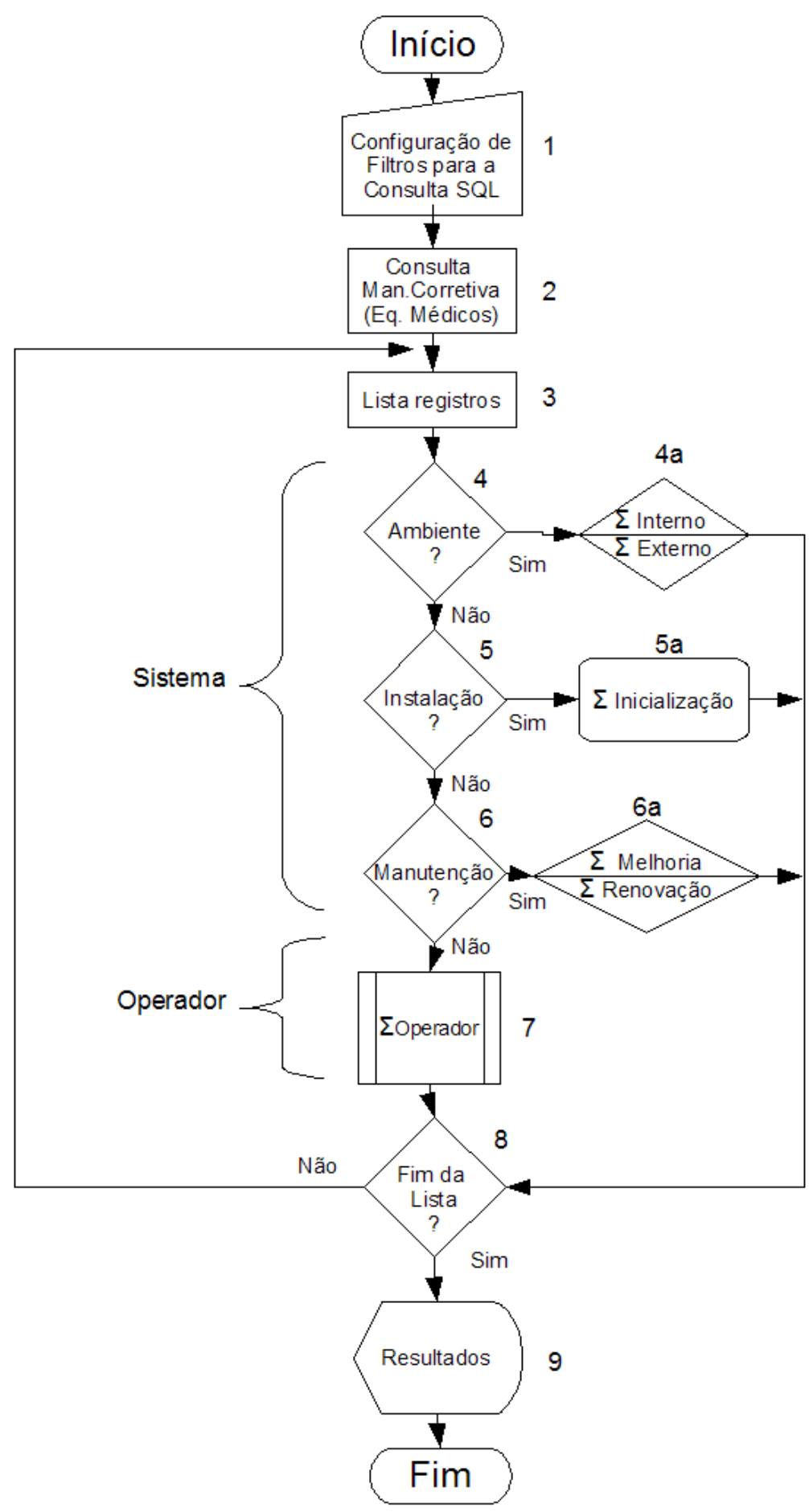

Fonte: Elaborado pelos autores. 
FIGURA 3 - Energia do operador ao longo da jornada semanal

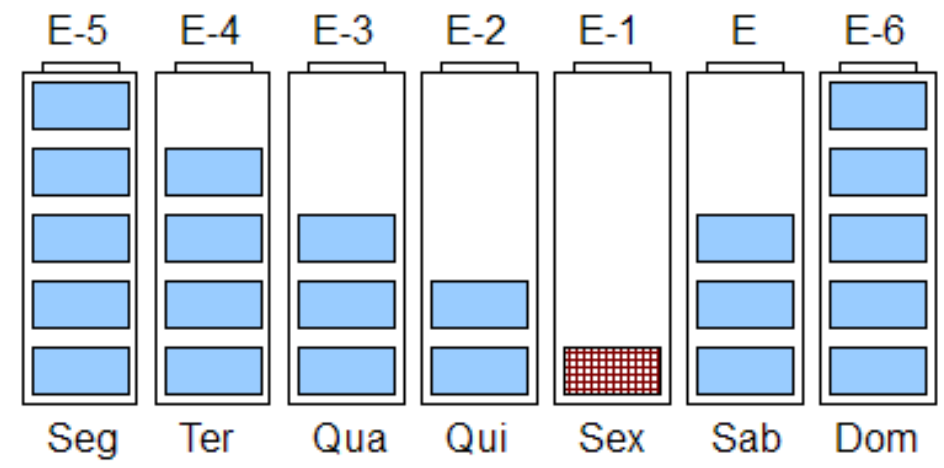

Fonte: Elaborado pelos autores.

Pereira $(2014$, p. 72$)$ aponta, de forma corroborativa a teoria de acidente baseada em energia, que as consequências da falta de concentração e atenção provocada pelo esgotamento da energia (Burnout) são a predisposição a acidentes e a baixa produtividade. Logo, pela observação da figura 3, é possível deduzir que quanto menor o nível de energia do operador, maior a chance de erros de operação do equipamento médico e, com isso, maior número de registros de manutenção serão contabilizados. Desta forma, o protótipo de software pode criar vetor com a indexação proposta para contabilizar os registros de acordo com o nível de energia. Contudo, tendo em vista o estudo de Poltosi e Gómez (2007) para escalas de trabalho, diante de um feriado que possa ser motivo para tornar o plantão impopular, e supondo ser este o evento de descanso desejado pela maioria dos operadores no ato do planejamento da escala de trabalho, então E deve ser atribuído ao inicio do feriado prolongado, sendo reaplicada a regra de indexação para indicar menores níveis de energia nos dias que antecedem o início do descanso. Desta forma, o protótipo de software proposto por este estudo poderia se aproximar das regras que delimitaram o planejamento da escala de trabalho estimando a alteração na jornada a partir dos feriados nacionais, por exemplo, reduzindo assim distorções de classificação quanto a energia do operador na etapa 7.

\section{ANÁLISE DOS RESULTADOS}

O protótipo de software foi aplicado aos registros de manutenções ocorridos entre 2012 e 2014, em dois hospitais que atendem ao SUS e que não compartilham operadores por estarem geograficamente distantes, denominados A e B. Ao todo foram analisados 1.773 registros de manutenção corretiva de equipamentos médicos relacionados aos centros cirúrgicos (CC) e de terapia intensiva (CTI), sendo que o hospital A apresentou 1553 registros e o hospital B, 220 registros. Ambos os hospitais estão localizados no estado de Minas Gerais e detêm um conjunto de equipamentos médicos com elementos comuns, denotando mesmo nível de tecnodependência. Contudo, o hospital A tem aproximadamente o dobro do quantitativo de salas cirúrgicas e leitos de terapia intensiva que $\mathrm{B}$, ou seja, o número de equipamentos médicos também é maior que o existente no hospital B.

Assim sendo, o hospital A foi escolhido para a análise em nível micro pelo maior número de registros. O protótipo de software classificou 36\% dos registros como atribuído ao componente 
Operator, e 64\% restantes como sendo de outros componentes. Considerando equipamentos de anestesia no centro cirúrgico, o software encontrou 44 registros, distribuídos conforme gráfico 1:

GRÁFICO 1 - Classificação no hospital A para equipamentos de anestesia

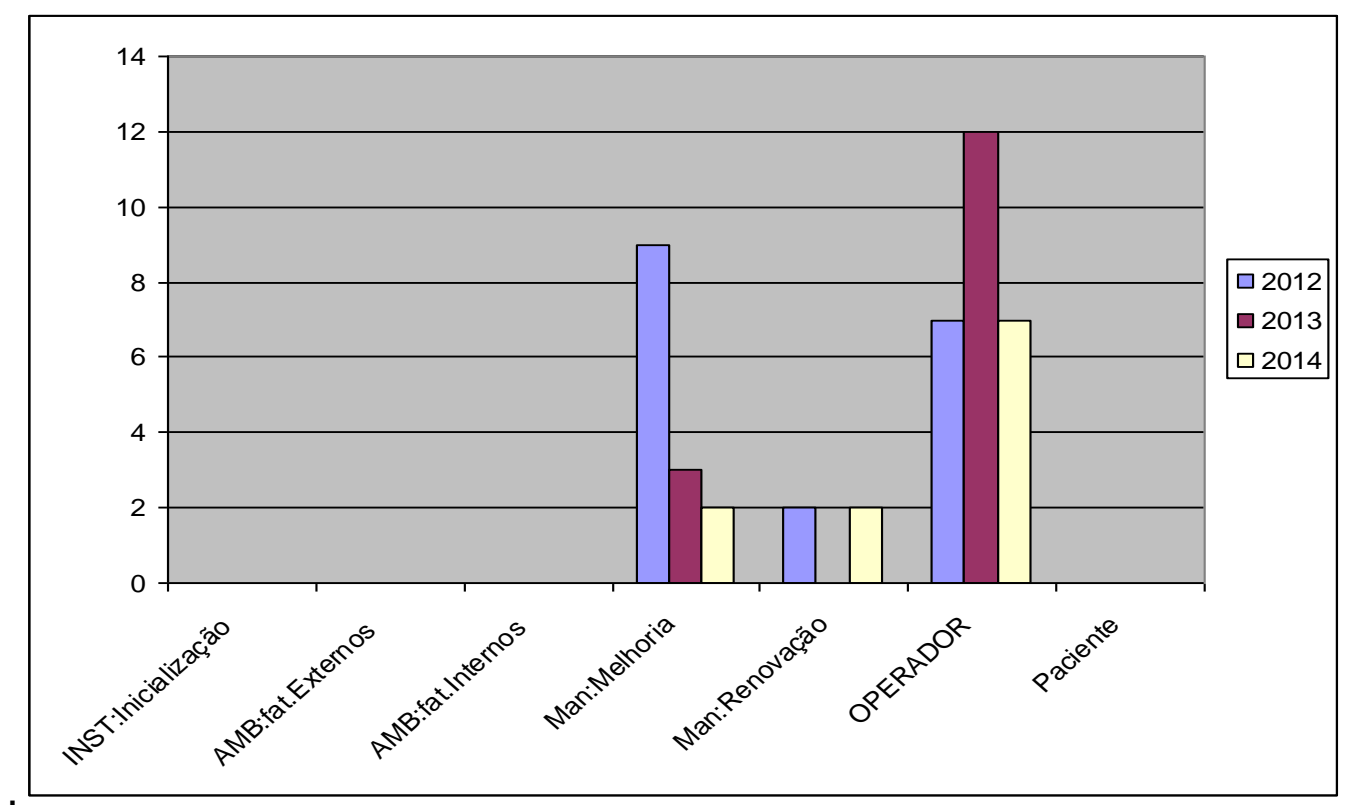

Fonte: Dados de pesquisa.

O componente Operator, a partir de 2012, ano no qual houve a detecção de necessidade de melhoria em manutenção, se mostra como o componente que detém a maior parte dos registros. A distribuição dos registros ao longo da jornada de trabalho semanal pode ser vista no gráfico 2, onde fica perceptível que o equipamento médico é utilizado satisfatoriamente no inicio da jornada. No entanto, ao final da jornada o número de registros de manutenção aumenta.

GRÁFICO 2 - Componente Operador para equipamento de anestesia do hospital A

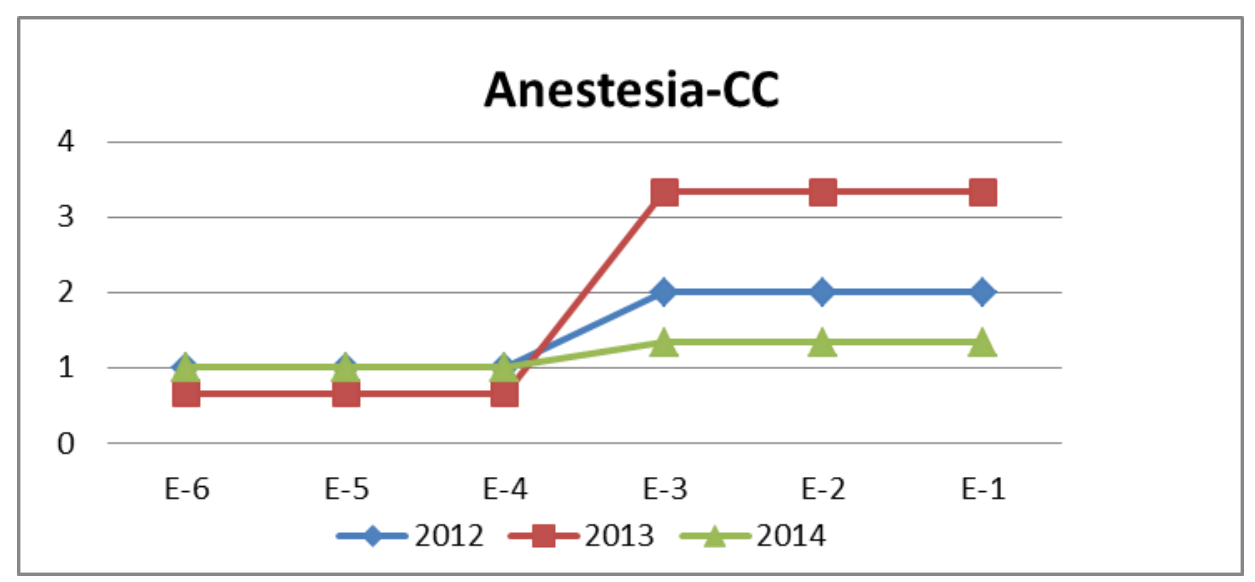

Fonte: Dados de pesquisa. 
Apesar dos resultados do software entre os anos de 2012 e 2014 corroborarem a confirmação da síntese gráfica como indicadora de estresse, a análise retrospectiva manual dos relatos contidos nos registros foi necessária para averiguar se houve erro de classificação que atribuísse registros indevidamente ao Operador, mas cujas causas-raízes se encontram em outros componentes. Desta forma, a análise encontrou um número reduzido de erros de classificação por parte de preenchimento incorreto da ordem de serviço pelo técnico da engenharia clínica do hospital A e também apontou, em grande parte, erros repetitivos dos operadores, como vazamentos por descuidos de montagem, falhas na verificação de funcionamento, perda de desempenho por falta de limpeza, etc. $\mathrm{O}$ ciclo dos erros se perpetua ano após ano, não porque não houve o aprendizado dos profissionais de assistência mais experientes pela observação das soluções apresentadas no passado aos mesmos problemas, mas pela precariedade da comunicação e do planejamento dos supervisores envolvendo a equipe de Operadores dentro da cultura organizacional, o que torna as tarefas desempenhadas, principalmente pelos profissionais residentes, mais estressantes.

Ainda no hospital A, foram analisados pelo software proposto 233 registros de manutenção corretiva em bombas de infusão do centro de terapia intensiva de pacientes adultos, sendo que falhas atribuídas ao componente Operador representaram 34,3\%, estando em concordância com Amoore (2014), que relatou em seus estudos valores acima de $30 \%$. A classificação obtida pelo software para bombas de infusão pode ser vista pelo Gráfico 3, onde se percebe problemas de manutenção em 2012, a introdução de novos equipamentos médicos em 2013, e, finalmente, maior percepção de erros de operação no ano de 2014 apesar da realização de treinamento documentado.

GRÁFICO 3 - Classificação de bombas de infusão no hospital A

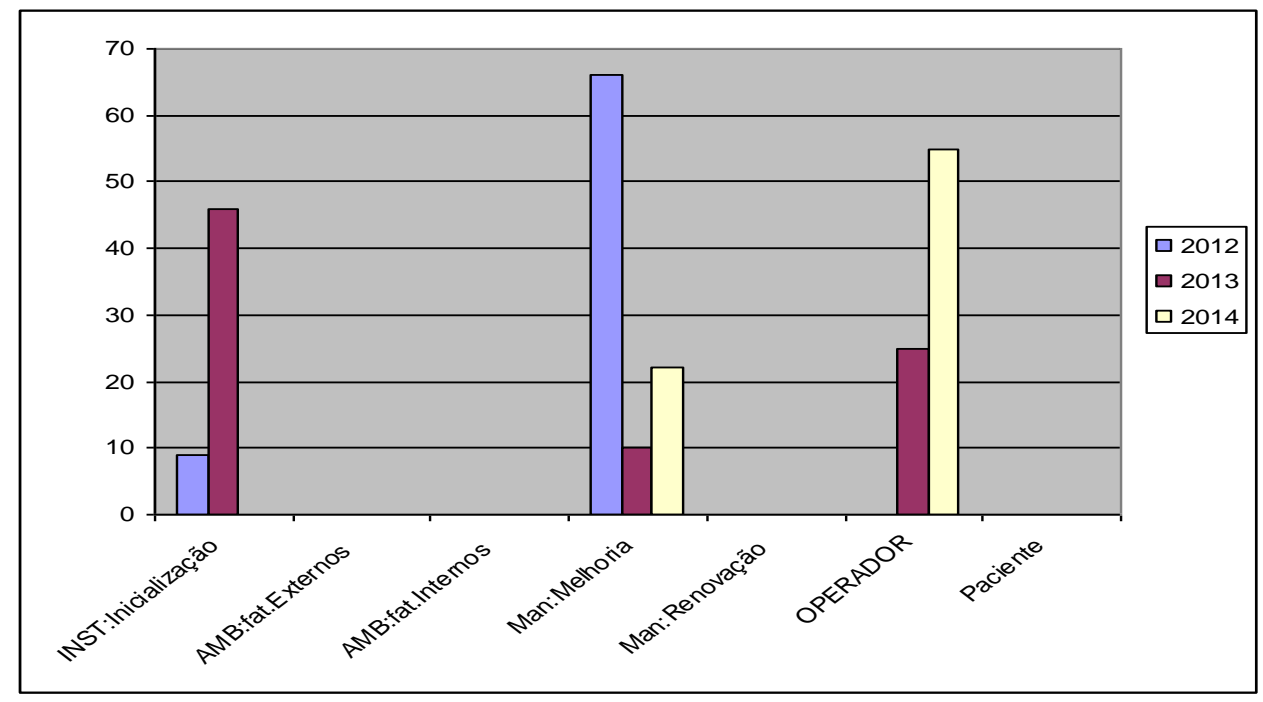

Fonte: Dados de pesquisa.

O Gráfico 4 mostra que em 2014 a equipe de enfermagem estava sob estresse. 
GRÁFICO 4 - Componente Operador para equipamento "bomba de infusão" do hospital A

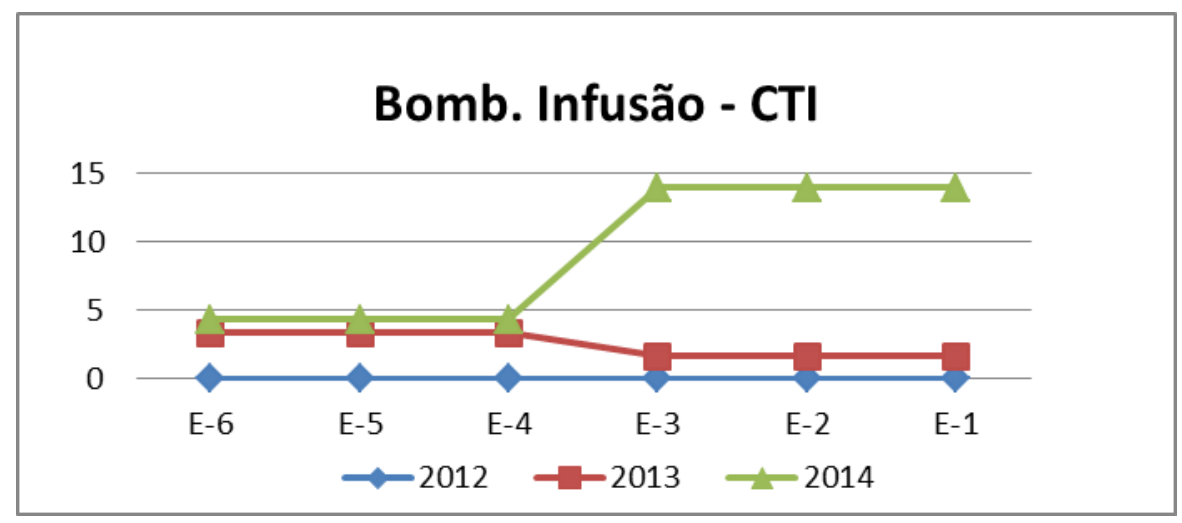

Fonte: Dados de pesquisa.

Em nível macro, considerando apenas o ambiente centro cirúrgico do hospital A e do hospital B, sem indicar o tipo de equipamento médico na configuração do protótipo de software, é obtido o perfil de estresse de toda a população de operadores pela soma das interações com os diversos equipamentos médicos disponibilizados naquele ambiente. A partir da comparação dos gráficos 5 e 6, é possível afirmar que, dado o número de registros e a estabilidade das médias obtidas, o hospital B oferece ambiente menos propício ao estresse que A.

GRÁFICO 5 - Ambiente CC no hospital A, registros por jornada semanal

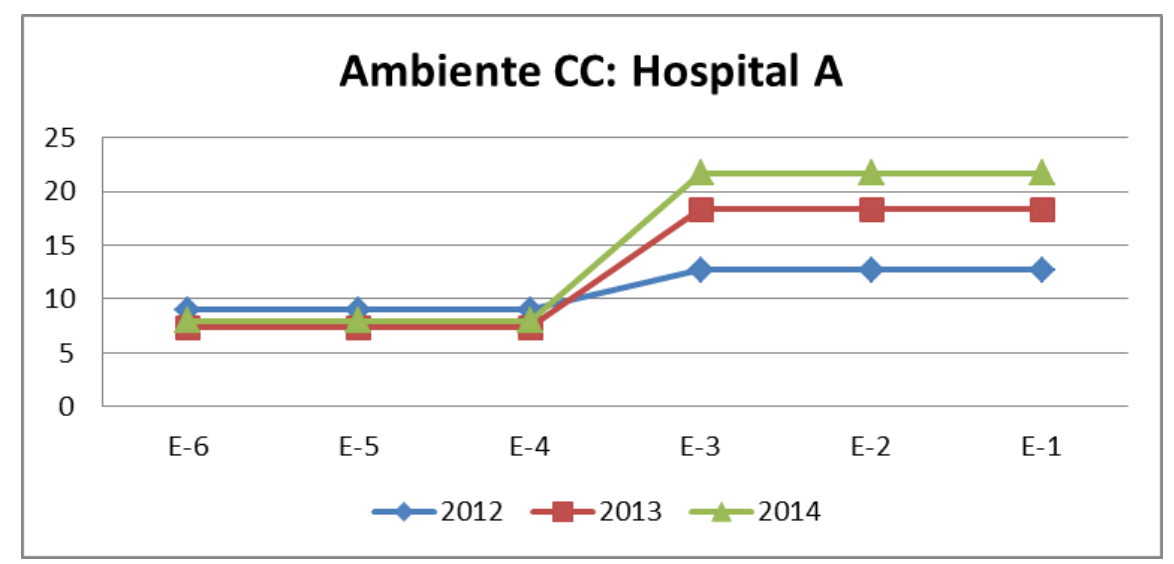

Fonte: Dados de pesquisa. 
GRÁFICO 6 - Ambiente CC no hospital B, registros por jornada semanal

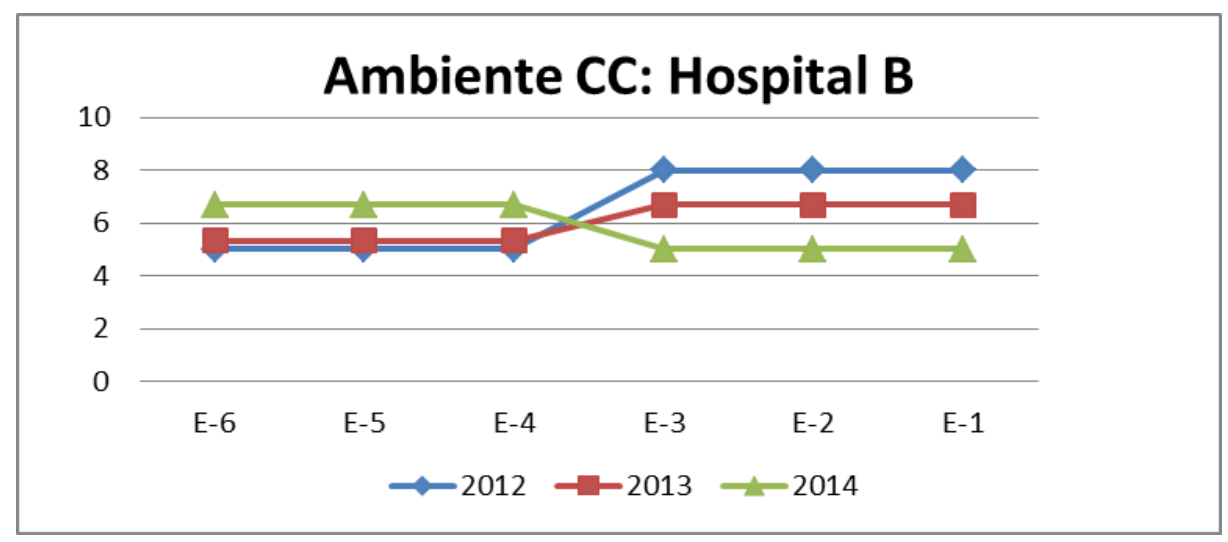

Fonte: Dados de pesquisa.

Apesar do maior porte do hospital A em relação ao B, percebe-se que, no inicio da jornada de trabalho, ambos possuem médias abaixo de 10 registros, mas somente o hospital B consegue manter este limite até o fim da jornada. Resultado semelhante foi observado em centros de terapia intensiva. Isso significa que o profissional de saúde do hospital B teria mais energia e cometeria menor número de erros, impactando positivamente na qualidade dos serviços prestados aos pacientes.

\section{CONCLUSÃO}

Este estudo propôs, através de engenharia de software, a aplicação computadorizada de síntese gráfica para aproveitamento dos registros de manutenção existentes em um sistema de informação de engenharia clínica para diversas aplicações: primeiramente, para a gestão de risco, com ênfase na detecção dos indícios e prevenção do agravamento de estresse e de erros que resultassem em eventos adversos; posteriormente, para a desmistificação sobre a eficácia do treinamento de operação como solução definitiva em ambiente de centros cirúrgicos e de terapia intensiva como forma de evitar danos na interface homem-máquina ocorridos ao longo da jornada semanal de trabalho e da vida útil do equipamento médico. E, enfim, para melhoria da qualidade nos serviços de saúde.

Para o gestor em saúde pública e stakeholders, o software demonstrou que pode ser útil como meio de diagnosticar o comportamento de risco e promover a melhoria contínua fomentando participação focada dos profissionais de saúde na resolução de problemas em segundo nível, ou em double loop, o que oferece maior solidez para, por exemplo, empregar recursos financeiros em novas incorporações.

Entretanto, apesar das evidências coletadas ao longo do ensaio da versão de software, o estudo encontrou limitações, que dificultaram a obtenção de melhores resultados e, portanto, podem demandar novas pesquisas. Dentre as limitações, está o fato de não ser possível comparar, no mesmo período analisado, estudos científicos já publicados com o uso do MBI nos hospitais A e $\mathrm{B}$; e, ainda, pelo fato de ser uma análise documental, não foi possível revisar o formulário (ordem de serviço de manutenção corretiva implantada conforme ANVISA (2010, p. 298)) de forma a adequar a coleta de dados e reduzir o equívoco de preenchimento por parte do técnico de engenharia clínica em alguns casos. Outra limitação consistiu da ausência de informações acerca de recessos, exatidão de início e fim de férias, faltas e feriados municipais. Tal lacuna pode ter 
reduzido a precisão da classificação e o consequente cálculo das médias do número de ocorrências de erros atribuídos aos operadores. Ainda, o estudo encontra limitação pela natureza dos hospitais, que são ligados ao Sistema Único de Saúde brasileiro. É possível que os resultados aqui apresentados não se estendam integralmente aos hospitais particulares da rede suplementar, exclusivos ao atendimento de planos de saúde privados.

Por fim, o protótipo de software fruto deste estudo é promissor a ponto de se tornar objeto de registro de propriedade intelectual via universidade: sua aplicação prática e sincera dentro dos estabelecimentos assistenciais de saúde permitirá que seus resultados instiguem o diálogo nas equipes assistenciais em busca de soluções mais duradouras e sustentáveis em seus ambientes de trabalho, com benefícios mais tangíveis aos pacientes. Soluções que implicarão maior confiança e qualidade em múltiplas dimensões do serviço de saúde, em um ciclo de provável coexistência colaborativa e virtuosa entre a tecnologia e o ser humano.

\section{REFERÊNCIAS}

AMOORE, J. N. A Structured Approach for Investigating the Causes of Medical Device Adverse Events. Journal of Medical Engineering, 2014.

ANVISA. Manual de Tecnovigilância: Abordagens de Vigilância Sanitária de Produtos para a Saúde Comercializados no Brasil. Brasília: Ministério da Saúde, 2010.

ANVISA. Assistência Segura: Uma reflexão teórica aplicada à prática. Brasília: Ministério da Saúde, 2013.

AQUINO, J. M. Estressores no trabalho de Enfermeiras em Centro Cirúrgico: consequências pessoais e profissionais. Ribeirão Preto: Escola de Enfermagem de Riberão Preto da Universidade de São Paulo, 2005.

BURKE, E. K. et al. The State of the Art Of Nurse Rostering. [S.1.]: [s.n.]. 2004. p. 441-499.

CALIL, J. S.; GOMIDE, E. T. Equipamentos médico-hospitalares e o gerenciamento da manutenção - capacitação a distância. Brasília: [s.n.], 2002.

DEITEL, H. M.; DEITEL, P. J. Como programar em C. Rio de Janeiro: LTC, 1999.

DeROSIER, J. et al. Using Health Care Failure Mode and Effect AnalysisTM: The VA National Centre for Patient Safety's Prospective Risk Analysis System. Journal on Quality Improvement 28(5): p. 248-67, 2002.

DYRO, J. The Clinical Engineering Handbook. Burlington: Elsevier Science and Technology Books, 2004.

ELIAS, G. A. Metodologia para avaliação de parâmetros ambientais em serviços de saúde. Campinas: Unicamp, 2013.

FERREIRA, M. J. Saúde no Trabalho - Temas básicos para os profissionais que cuidam dos trabalhadores.São Paulo: Roca, 2000. 
GLOWACKI, L. A. Avaliação de efetividade de sistemas concentradores de oxigênio: uma ferramenta em gestão de tecnologia médico-hospitalar. Dissertação (mestrado) -, Universidade Federal de Santa Catarina, Florianópolis, 2003. 142 p.

HERMINI, A. H. A tecnologia e sua relação com os procedimentos assistenciais de saúde. In: BURMESTER, H. Gestão de materiais e equipamentos hospitalares. São Paulo: Saraiva, 2013.

LIMA, F. D. et al. Síndrome de Burnout em Residentes da Universidade Federal de Uberlândia, Uberlândia, p. 137-146, 2007.

MARTINS, L.; FRAGATA, J. O erro em Medicina - perspectivas do indivíduo, da organização e da sociedade. 4. ed. Coimbra: Almedina, 2014.

MASLACH, C.; JACKSON, S. E.; LEITER, M. P. Maslach Burnout Inventory Manual. Palo Alto: Consulting Psychologist Press, 1986.

MASLACH, C.; JACKSON, S. E.; LEITER, M. P. Job Burnout. Annual Review of Psychology, n. 52, p. 397-422, 2001.

PAFARO, R. C.; MARTINO, M. M. F. Estudo do estresse do enfermeiro com dupla jornada de trabalho em um hospital de oncologia pediátrica de Campinas. Revista Escola de Enfermagem da USP, Campinas, v. 38, p. 152-160, 2004.

PEREIRA, A. M. T. B. Burnout - quando o trabalho ameaça o bem-estar do Trabalhador. 4. ed. São Paulo: Casa do Psicólogo, 2014.

POLTOSI, M. R.; GÓMEZ, A. T. Elaboração de escalas de trabalho de técnicos de enfermagem com busca Tabu e Algorítimo Genético. SBPO, 39., Fortaleza, [s.n.], 2007.

REASON, J. Understanding adverse events: human factors. Quality in Health Care, n. 4, p. 8089, 1995.

SÁ, R. A. M. Levantamento e análise de eventos adversos com aparelhos de tomografia computadorizada. Rio de Janeiro: Fundação Oswaldo Cruz, 2009.

SANTOS, C. L. M. E. A. Fatores de estresse na atividade de médicos em João Pessoa. Produção, v. XXI, n. 1, p. 181-189, 2011.

SHEPHERD, M. Systems Approach to Medical Device Safety. In: DYRO, J. F. The Clinical Engineering Handbook. Burlington: Elsevier Science and Technology Books, 2004. p. 246-249.

TUCKER, A. ; EDMONDSON, A. Why hospitals don't learn from failures: Organizational and psychological dynamics that inhibit system change. California Management Review, v. 45, n.2, p. 55-72, 2003.

WESTIMAN, M; EDEN, D. Effects of a respite from work on burnout: Vacation relief and fadeout. Journal of Applied Psychology, n. 82, p. 516-527, 1997.

WU, J.; ZHAO, T. C-HFAMF: A new way to Accident Analysis considering Human Factor. Beijing: Beijing University of Aeronautics and Astronautics, 2011. 